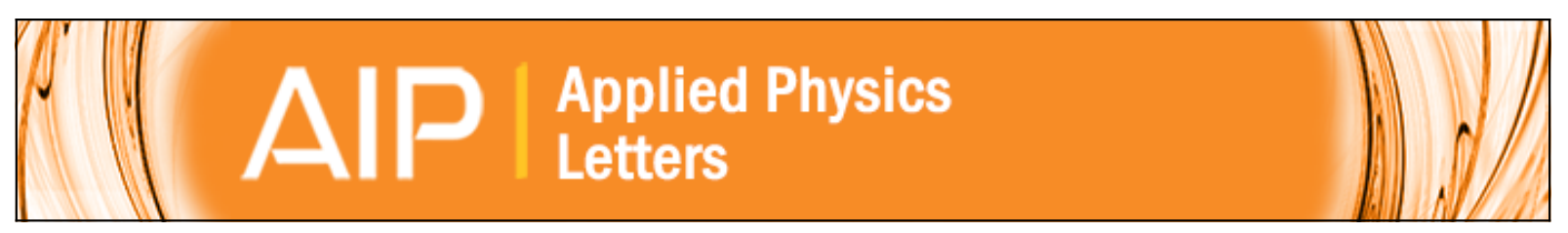

\title{
A thermal logic device based on fluid-solid interfaces
}

Sohail Murad and Ishwar K. Puri

Citation: Applied Physics Letters 102, 193109 (2013); doi: 10.1063/1.4807173

View online: http://dx.doi.org/10.1063/1.4807173

View Table of Contents: http://scitation.aip.org/content/aip/journal/apl/102/19?ver=pdfcov

Published by the AIP Publishing

\section{AlP Re-register for Table of Content Alerts}




\title{
A thermal logic device based on fluid-solid interfaces
}

\author{
Sohail Murad and Ishwar K. Puria) \\ Department of Chemical Engineering, University of Illinois at Chicago, Chicago, Illinois 60607, \\ USA and Department of Engineering Science and Mechanics, Virginia Tech, Blacksburg, Virginia 24061, USA
}

(Received 25 February 2013; accepted 4 May 2013; published online 17 May 2013)

\begin{abstract}
Thermal rectification requires that thermal conductivity not be a separable function of position and temperature. Investigators have considered inhomogeneous solids to design thermal rectifiers but manipulations of solid lattices are energy intensive. We propose a thermal logic device based on asymmetric solid-fluid resistances that couples two fluid reservoirs separated by solid-fluid interfaces. It is the thermal analog of a three terminal transistor, the hot reservoir being the emitter, the cold reservoir the output, and smaller input reservoirs as the base. Changing the input temperature alters the transport factor and the flux gain as does the base current in a transistor. (C) 2013 AIP Publishing LLC. [http://dx.doi.org/10.1063/1.4807173]
\end{abstract}

Thermal rectification is a key requirement for any thermal logic device. Mathematically, a sufficient condition for rectification is that the thermal conductivity $k$ (or inverse resistance $1 / R$ ), which is intrinsically a function of position and temperature, is not functionally separable, i.e., $k(x, T) \neq$ $f(x) g(T)$. Because this inequality is satisfied for inhomogeneous or asymmetric materials, investigators have almost exclusively designed thermal logic devices using inhomogeneous solids. The fixed structures of solids can also be tuned by applying mechanical stress. ${ }^{1,2}$ Since incompressible fluids are generally homogeneous, these have been typically ignored for such use. Often it is important to be able to tune such devices for specific applications. However, structural manipulations of solid lattices are much more energy intensive than methods to change the structure that alter the wettability of solid-liquid interfaces. ${ }^{3-6}$ For instance, in a solidfluid system, altering the cold-side thermal resistance by changing surface wetting leads to thermal rectification., Changing the interfacial resistance adjacent to the hot wall or the overall bulk thermal resistance also produces rectification although these changes are not as effective. ${ }^{3}$ Here we propose a thermal logic device based on asymmetric solidfluid resistances. The resulting device is similar in principle to an electronic circuit containing interconnected components with disparate electrical resistances.

The ability to control thermal transport without changing its driving potential, i.e., the temperature difference across the system, opens up the possibility of designing innovative devices based on thermal logic. By employing interconnected thermal rheostats, diodes, and gates that control the direction and magnitude of heat transfer, thermal logic devices could transport data using $\mathrm{GHz}$ frequency phonon thermal currents. These devices could enhance energy conservation, e.g., through better solar energy collectors, energy-efficient buildings, and appliances such as refrigerators, lead to thermal digital computers that use phonons rather than electron transport, and improve the thermal management of power systems. ${ }^{8-10}$ They could use the waste heat from electronic devices, producing significant power savings. By taking advantage of the nonlinearity of the

a)ikpuri@vt.edu lattice dynamics that influences thermal conductivity, thermal logic devices could be made strongly nonlinear and therefore more tunable. ${ }^{11}$

While hypothetical thermal logic devices based on thermal control that contain similar elements to electronic logic have been discussed, ${ }^{6,7,9,12}$ the fabrication of thermally based diodes, transistors, and logic circuits has remained elusive. The fundamental problem is that, unlike electrons that are point particles with fixed properties, heat carrying phonons are diffuse energy carriers whose properties are changeable. ${ }^{13}$ This is a seemingly intractable issue at the macroscale. However, the nanoscale provides intriguing possibilities for thermal logic by manipulating phonon transport. ${ }^{12}$ Since mass disorder alters the thermal conductivity of a nanomaterial, ${ }^{13}$ mass reorganization can also lead to a varying thermal conductivity. This can be exploited to devise thermal rectification, ${ }^{14}$ e.g., through a thermal diode, where the heat flux is directionally dependent. ${ }^{3,4,12,15-17}$ Complete rectification allows the material to behave both as a conductor and an insulator depending upon the direction of heat transfer.

A temperature difference $\Delta T$ that produces a thermal flux $q$ overcomes the system resistance $R=\Delta T / q>0$. With rectification, $q$, hence $R$, depends upon the direction in which the gradient $(\Delta T / \Delta x)$ is imposed. Consequently, $q_{l \rightarrow r} \neq q_{r \rightarrow l}$, where $l$ and $r$ denote the left and right faces of the material. ${ }^{3}$ To design a thermal analogy to a semiconductor device, such as the tunnel diode, $k(x, T)$ must be manipulated to decrease $q$ with increasing $\Delta T$ and thus produce a negative differential thermal resistance $\Delta R{ }^{6,12,18}$ For the same hot and cold temperatures, respectively, $T_{h}$ and $T_{c}$, such a device will have multiple stable steady states.

Our conceptual thermal logic device couples three fluid reservoirs that are separated by two walls, as illustrated in Fig. 1. The system is periodic so that the fluid between interfaces 1 and 4, moving from left to right, is essentially contained in the same reservoir, as are the fluids in the top and bottom reservoirs (above and below the walls). In the middle of each reservoir, a thin volumetric fluid layer is maintained at fixed temperatures on the hot and cold sides, respectively, $T_{h}$ or $T_{c}$. This facilitates the heat fluxes $q_{h}$ and $q_{c}$ in these hot and cold reservoirs, as shown in Fig. 1(a). The two transverse ends of each wall are coupled with smaller fluid reservoirs, 


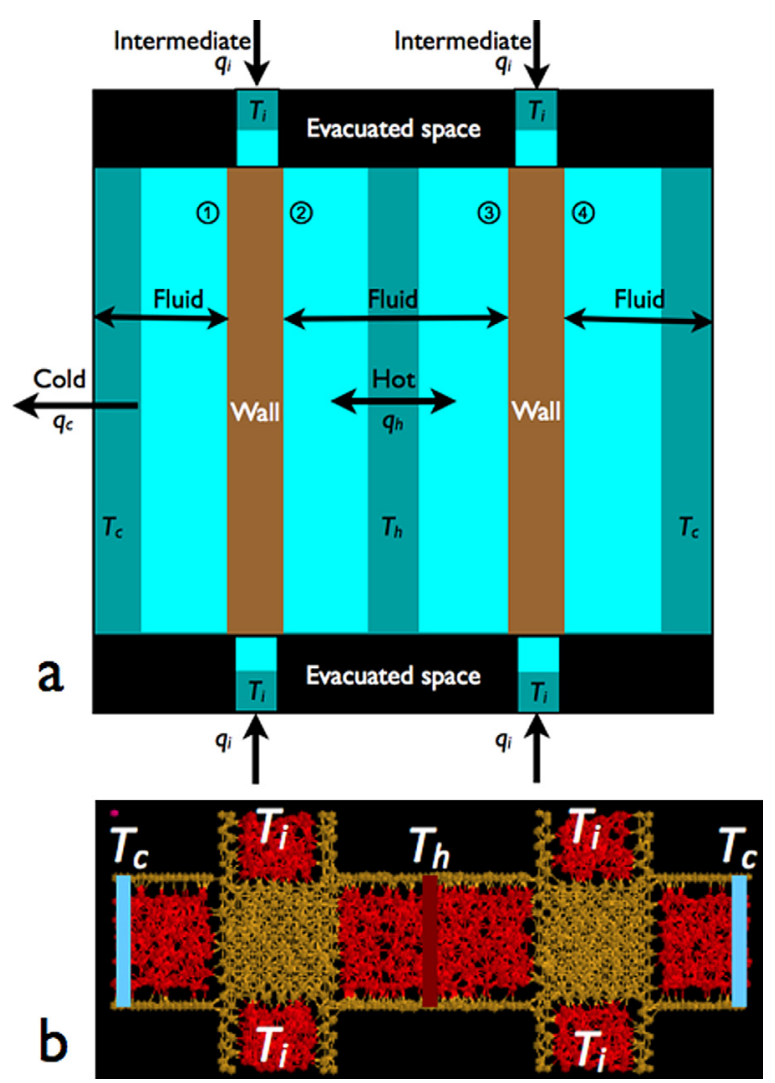

FIG. 1. (a) The thermal logic device couples two fluid reservoirs containing equal volumes of fluid that are separated by two walls. In the middle of each reservoir, a thin volumetric fluid layer is maintained at a fixed temperature that facilitates the heat fluxes $q_{h}$ and $q_{c}$ in these hot and cold reservoirs. The two ends of each wall are coupled with smaller fluid reservoirs at the same temperature $T_{i}$, which are adjusted to facilitate the variable heat flux $q_{i}$. Asymmetry is introduced by independently changing the wettability of the four interfaces separating the two reservoirs. (b) The fluid regions in which the temperature is controlled are shaded solid red (hot) and blue (cold), water molecules are shaded as red spheres, while the solid and confining walls are yellow spheres. The system consists of 1200 molecules of water and 1696 of silicon.

all at the same temperature $T_{i}$, which can be adjusted to facilitate a variable heat flux $q_{i}$. When the top and bottom boundaries of the system shown in Fig. 1(a) are adiabatic, then $q_{i}=0$. Asymmetry can be introduced by independently changing the wetting behaviors of the four interfaces provided by the walls separating the two reservoirs. From energy conservation, $q_{h}+q_{i}=q_{c}$.

The molecular dynamics (MD) simulations consider thermal transport across hot and cold fluid regions that enclose a bulk fluid and a solid wall whose surface can be manipulated to make it hydrophobic (neutral) or hydrophilic (charged). The system setup is shown in Figure 1(b). The fluid regions in which the temperature is controlled are shaded solid red (hot) and blue (cold), water molecules are shaded as red spheres, while the solid and confining wall molecules are yellow. The walls influence small portions of the fluid adjacent to them, but the remaining fluid in the reservoirs exhibits bulk properties where the water density $\rho \sim$ $980 \mathrm{~kg} / \mathrm{m}^{3}$. The system consists of 1200 molecules of water and 1696 of silicon. All Si atoms are tethered to their equilibrium sites and allowed to vibrate harmonically. The molecules in the system are provided with initial Gaussian velocity distributions and the hot and cold fluid regions are imparted average temperatures $T_{h}=1209 \mathrm{~K}$ and $T_{c}=403 \mathrm{~K}$ with a Gaussian thermostat, while the temperature of the input regions $T_{i}$ varies between 403 and $1209 \mathrm{~K}$. A Gaussian thermostat is more efficient for nonequilibrium configurations and in the thermodynamic limit its linear response is identical to that of the Nose-Hoover thermostat. ${ }^{19,20}$ This arrangement results in an globally averaged fluid temperature $\left(T_{h}+T_{c}\right) / 2 \sim 806 \mathrm{~K}$. (The actual average varies slightly depending upon $T_{i}$.) At this average temperature, the bulk fluid is supercritical. These relatively high wall temperatures are conducive for our simulations since they facilitate larger heat transfer rates and minimize data scatter. However, the fundamental aspects of rectification apply even at lower temperatures and remain unaltered. ${ }^{3,4}$ The $(N, V, T)$ simulations proceed with uniform step sizes of 1 fs and are allowed to equilibrate. Results are reported when the simulations have progressed by $2 \times 10^{6}$ time steps and reached a steady state. Longer simulations have confirmed the accuracy and stability of the temperature and density profiles.

The MD algorithm ${ }^{3,4}$ uses the quaternion method. Intermolecular interactions follow the potential model $u_{i j}=4 \varepsilon_{i j}\left(\left(\sigma_{i j} / r_{i j}\right)^{12}-\left(\sigma_{i j} / r_{i j}\right)^{6}\right)+\left(Q_{i} Q_{j}\right) / r_{i j}$, where $\sigma_{i j}$ and $\varepsilon_{i j}$ denote the L-J interaction parameters, $r_{i j}$ the scalar distance between sites $i$ and $j$ (we note that water includes $2 \mathrm{H}$ sites and one $\mathrm{O}$ site water to account for the orientation of water), and $Q_{i}$ and $Q_{j}$ the charges on these sites. Depending on the type of wall surface that is desired, $Q_{i}$, or $Q_{j}$ can be set to zero or imparted the values $\pm \delta$ to make a surface hydrophilic. The simple-point-charge (SPC) potential is used, since it realistically represents water properties, including the thermal conductivity for high density and supercritical fluids. ${ }^{21} \mathrm{Si}$ is modeled with an L-J potential and three-body interactions are indirectly enforced for its coordinated tetrahedral bonded structure by tethering these atoms to their equilibrium sites. Cross interactions are modeled using Lorentz-Berthelot mixing rules, and the reaction field method replicates long-range interactions. A cutoff distance of $9.5 \AA$ is used. The system is $\sim 12 \mathrm{~nm}$ long and $4.25 \mathrm{~nm}$ wide with a depth (in the plane of Fig. 1(a)) of $2 \mathrm{~nm}$. Periodic boundary conditions are applied.

When all four faces are inert, i.e., $Q_{i}=0$ for each of the four walls, and $T_{i}$ is varied, the fluxes adjust linearly to the specified values of $T_{h}$ and $T_{c}$, as shown in Fig. 2 in reduced units. As $T_{i}$ increases, so do $q_{c}$ and $q_{i}$ whereas $q_{h}$ decreases. This allows us to identify three regions where (1) $q_{h}>q_{c}$, (2) $q_{h}<q_{c}$ and $q_{i}<q_{c}$, and (3) $q_{h}>q_{i}$. From energy conservation, $q_{h}+q_{i}=q_{c}$, i.e., the amplification of the hot side flux for this device is $\alpha^{*}=\left|\partial q_{h} / \partial q_{i}\right|=\left|\partial q_{c} / \partial q_{i}-1\right|$. Since from Fig. 2, $\partial q_{c} / \partial q_{i}>0, \alpha^{*}<1$ always for this device. The corresponding temperature profiles are illustrated in Fig. 3. These show how the system adjusts to changing $T_{i}$. The response of the fluid reservoirs to changes in this temperature is more dynamic than that of the solid walls. The temperature discontinuities that are representative of the interfacial Kapitza resistances associated with $q_{h}$ and $q_{c}$ are apparent at both the hot and cold solid-fluid interfaces. ${ }^{14,22-24}$ Increasing the value of $T_{i}$ increases that of $R$ for the hot wall but decreases this solid-fluid resistance at the cold wall.

The nature of a nanoscale interface significantly influences thermal transport through the combined effect of the 


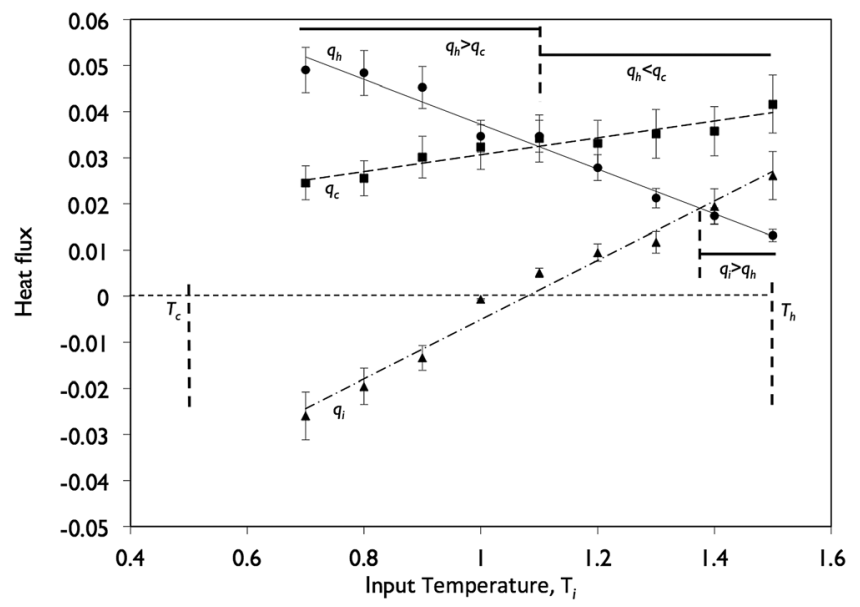

FIG. 2. The variation in the hot, cold, and input heat fluxes $q_{h}, q_{c}$, and $q_{i}$ with changing input temperature $T_{i}$. When all four faces are inert and $T_{i}$ is varied, the fluxes adjust linearly to the specified values of the hot and cold temperatures $T_{h}$ and $T_{c}$. The dimensionless temperatures should be multiplied by $806 \mathrm{~K}$ to recover the units of $\mathrm{K}$. Likewise, each unit of heat in reduced units corresponds to $4.25 \times 10^{-6} \mathrm{~W}$.

fluid- and solid-side resistances. ${ }^{23-25}$ Retaining the inert interfaces 2 and 4, as denoted in Fig. 1(a), but making interfaces 1 and 3 hydrophilic by imparting $\pm \delta$ charges to the corresponding walls, introduces asymmetry. The system now behaves differently from the interactions shown in Fig. 2 . This is illustrated through the nonlinear responses of $q_{h}, q_{c}$, and $q_{i}$ to $T_{i}$ (Fig. 4). In particular, there are now two regions where either $\left(\partial q_{h} / \partial T_{i}\right)$ or $\left(\partial q_{c} / \partial T_{i}\right) \approx 0$, which increases the value of $\alpha^{*}$ to at least unity. While the overall temperature profiles for a particular $T_{i}$ for this system are similar to those shown in Fig. 3, there are subtle differences related to the temperature discontinuities across the hot and cold walls that alter the corresponding values of $R$.

When $\left(\partial q_{h} / \partial T_{i}\right) \leq 0$, there is a near zero (or slightly negative) differential thermal resistance to heat flow, i.e., $q_{h}$

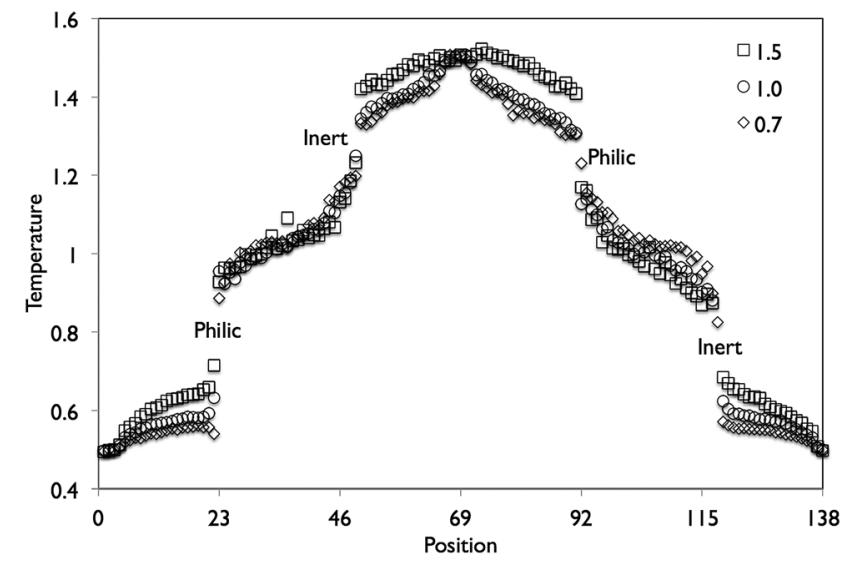

FIG. 3. The temperature profiles for the symmetric system show how the device adjusts to changing $T_{i}$. The response of the fluid to changes in this temperature is more dynamic than that of the $\mathrm{Si}$ walls. The temperature discontinuities representative of the interfacial Kapitza resistances associated with $q_{h}$ and $q_{c}$ are apparent at both the hot and cold solid-fluid interfaces. Increasing $T_{i}$ increases $R$ for the hot wall but decreases this solid-fluid resistance at the cold wall. The dimensionless temperatures should be multiplied by $806 \mathrm{~K}$ to recover the units of $\mathrm{K}$. The system dimension in the $\mathrm{x}$ direction is $12 \mathrm{~nm}$, which is divided into 138 slabs in each of which the local properties are averaged. The position numbers of these slabs are shown. The Si walls include slabs 24-49 and 93-117.

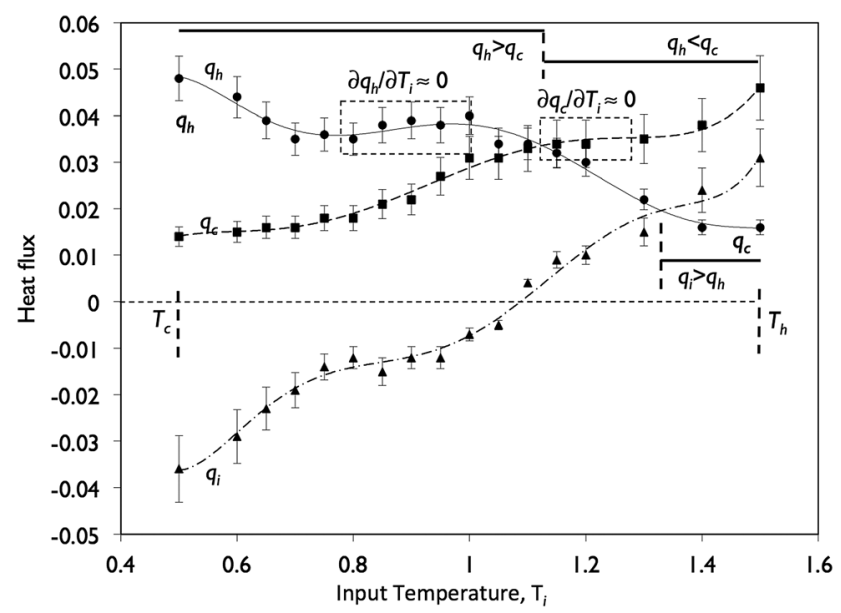

FIG. 4. When the interfaces 2 and 4 that are denoted in Fig. 1(a) are retained as inert and interfaces 1 and 3 are made hydrophilic, asymmetry is introduced. The device behaves differently in contrast to Fig. 2. The fluxes $q_{h}, q_{c}$, and $q_{i}$ have a nonlinear response to $T_{i}$. There are two regions now where either $\left(\partial q_{h} / \partial T_{i}\right)$ or $\left(\partial q_{c} / \partial T_{i}\right)$ is negligible. The dimensionless temperatures and the heat fluxes can be converted into dimensional units as previously discussed.

is virtually unchanged or slightly decreases with an increasing temperature difference $\Delta T=\left(T_{h}-T_{i}\right)$. This ability of the system to resist the potential for greater heat transfer enables it to behave as a logic device. ${ }^{6,7,9,12}$ Plotted differently in Fig. 5, the nonlinear response of the asymmetric system is illustrated through the relationship between $q_{h}$ and $q_{c}$ with $q_{i}$. There are distinct threshold fluxes, $q_{i, h}$ below which $\partial q_{h} / \partial q_{i}$ is again linear, and $q_{i, c}$ above which $\partial q_{c} / \partial q_{i}$ once again becomes linear. Both threshold fluxes enable the switching of $q_{h}$ and $q_{c}$ through a transition. The device shown in Fig. 1 is the thermal analog to the three terminal transistor where the temperature $T_{h}$ occurs due to the hot reservoir (emitter), $T_{c}$ at the output (or collector), and $T_{i}$ at the input (or base). Changing $T_{i}$ alters the transport factor $\alpha=q_{c} / q_{h}$ and the flux gain $\beta=q_{c} / q_{i}$ in a similar manner to the influence of the base current in a transistor.

These results demonstrate the conceptual feasibility of both thermal rectification and thermal logic in a fluid based

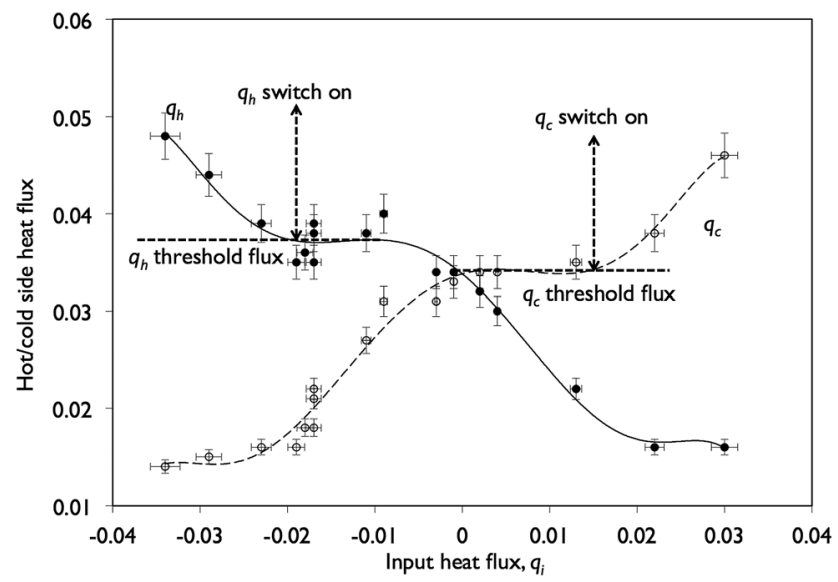

FIG. 5. The nonlinear response of the asymmetric system is observed through dependence of $q_{h}$ and $q_{c}$ on $q_{i}$. There are distinct threshold fluxes, $q_{i, h}$ below which $\partial q_{h} / \partial q_{i}$ is again linear, and $q_{i, c}$ above which $\partial q_{c} / \partial q_{i}$ once again becomes linear. Both threshold fluxes enable the switching of $q_{h}$ and $q_{c}$ through a transition. The dimensionless heat fluxes can be converted into dimensional units as discussed. 
device. Since it is simpler to alter the wettability of solidliquid interfaces than to produce inhomogeneous solids, our approach could be more feasible for realistic applications.

This research was supported by grants from the National Science Foundation (CBET 1246536/1246611).

${ }^{1}$ K. G. S. H. Gunawardana, K. Mullen, J. Hu, Y. P. Chen, and X. Ruan, Phys. Rev. B 85(24), 245417 (2012).

${ }^{2}$ M. Liangruksa and I. K. Puri, J. Appl. Phys. 109(11), 113501 (2011).

${ }^{3}$ S. Murad and I. K. Puri, J. Chem. Phys. 137(8), 081101-081104 (2012).

${ }^{4}$ S. Murad and I. K. Puri, Appl. Phys. Lett. 100(12), 121901-121905 (2012).

${ }^{5}$ M. Terraneo, M. Peyrard, and G. Casati, Phys. Rev. Lett. 88(9), 94302 (2002).

${ }^{6}$ L. Wang and B. Li, Phys. Rev. Lett. 101(26), 267203 (2008).

${ }^{7}$ B. Li, L. Wang, and G. Casati, Phys. Rev. Lett. 93(18), 184301 (2004).

${ }^{8}$ C. Chang, D. Okawa, H. Garcia, T. Yuzvinsky, A. Majumdar, and A. Zettl, Appl. Phys. Lett. 90(19), 193114 (2007).

${ }^{9}$ L. Wang and B. Li, Phys. Rev. Lett. 99(17), 177208 (2007).

${ }^{10}$ H. Tian, D. Xie, Y. Yang, T.-L. Ren, G. Zhang, Y.-F. Wang, C.-J. Zhou, P.-G. Peng, L.-G. Wang, and L.-T. Liu, Sci. Rep. 2, 523 (2012).
${ }^{11}$ M. E. Manley, presented at the MRS Proceedings: Symposium T Nanoscale Heat Transport-From Fundamentals to Devices, 2009.

${ }^{12}$ N. Li, J. Ren, L. Wang, G. Zhang, P. Hänggi, and B. Li, Rev. Mod. Phys. 84(3), 1045 (2012).

${ }^{13}$ G. Balasubramanian, I. K. Puri, M. C. Bohm, and F. Leroy, Nanoscale 3(9), 3714-3720 (2011).

${ }^{14}$ S. Murad and I. K. Puri, Chem. Phys. Lett. 476(4-6), 267-270 (2009).

${ }^{15}$ C. W. Chang, D. Okawa, A. Majumdar, and A. Zettl, Science 314(5802), 1121-1124 (2006).

${ }^{16}$ J. Hu, X. Ruan, and Y. P. Chen, Nano Lett. 9(7), 2730-2735 (2009).

${ }^{17}$ M. Hu, J. V. Goicochea, B. Michel, and D. Poulikakos, Appl. Phys. Lett. 95(15), 151903 (2009).

${ }^{18}$ Z. G. Shao, L. Yang, H. K. Chan, and B. Hu, Phys. Rev. E 79(6), 061119 (2009).

${ }^{19}$ D. J. Evans and B. L. Holian, J. Chem. Phys. 83, 4069 (1985).

${ }^{20}$ H. A. Posch and W. G. Hoover, in Molecular Liquids: New Perspectives in Physics and Chemistry, edited by J. J. C. Teixeira-Dias (Kluwer Academic Publishers, Dordrecht, 1992), pp. 527-547.

${ }^{21}$ F. Römer, A. Lervik, and F. Bresme, J. Chem. Phys. 137, 074503 (2012).

${ }^{22}$ P. L. Kapitza, J. Phys., USSR 4, 181 (1941).

${ }^{23}$ S. Murad and I. K. Puri, Appl. Phys. Lett. 92(13), 133105 (2008).

${ }^{24}$ S. Murad and I. K. Puri, Chem. Phys. Lett. 467(1-3), 110-113 (2008).

${ }^{25}$ G. Balasubramanian, S. Banerjee, and I. K. Puri, J. Appl. Phys. 104(6), 064306 (2008). 